Voix et Images

volxetimages

\title{
Notre première revue : l'Opinion publique (1870-1883)
}

\section{Jean-François Chassay}

Volume 9, numéro 2, hiver 1984

Roland Giguère

URI : https://id.erudit.org/iderudit/200444ar

DOI : https://doi.org/10.7202/200444ar

Aller au sommaire du numéro

Éditeur(s)

Université du Québec à Montréal

ISSN

0318-9201 (imprimé)

1705-933X (numérique)

Découvrir la revue

Citer cet article

Chassay, J.-F. (1984). Notre première revue : l'Opinion publique (1870-1883).

Voix et Images, 9(2), 131-142. https://doi.org/10.7202/200444ar d'utilisation que vous pouvez consulter en ligne.

https://apropos.erudit.org/fr/usagers/politique-dutilisation/ 


\section{Notre première revue: I'Opinion publique (1870-1883)}

\section{par Jean-François Chassay, Université de Montréal}

Présentée à sa parution comme «une revue essentiellement politique et littéraire», l'Opinion publique est intéressante à plus d'un titre. La qualité de ses illustrations, le choix de sés collaborateurs, la place dévolue à la littérature, l'analyse politique, tous ces éléments en font un journal de premier plan.

Cet hebdomadaire, le premier journal illustré canadien-français, paraît pour la première fois le premier janvier 1870. Grand in-folio de huit pages, il en aura douze à partir de janvier 1871. C'est Georges-Édouard Desbarats, homme d'affaires d'avant-garde, qui songe à lancer ce journal. Le brevet de la leggotypie, une variante de la lithographie, lui permet de reproduire à peu de frais des gravures. Il s'associe avec ceux qui en deviendront les premiers éditorialistes.

Le premier, Joseph-Adolphe Mousseau, est un avocat de 37 ans, assez connu, qui a publié une brochure très discutée, la Confédération, c'est le salut du Bas-Canada. Le second, Laurent-Olivier David, qui n'a que trente ans, s'est opposé violemment à la Confédération. Tous trois nationalistes, ils décident de fonder un périodique sans attache partisane, ni doctrine d'aucune sorte, qui défendrait les droits des francophones.

\section{IDÉOLOGIE}

Il est intéressant d'essayer de situer idéologiquement l'Opinion publique. ${ }^{1}$ Pour un journal qui semble de tendance libérale, il n'a rien d'une feuille anti-cléricale; les articles sur la célèbre affaire Guibord - le clergé versus l'Institut canadien - en font foi. Reproduire des articles contre la peine de mort est certes avant-gardiste pour l'époque, mais se concilie mal avec la publication de textes dithyrambiques sur Napoléon III, "grand démocrate». Et si la Commune de Paris (1870) se fait allègrement démolir, le lecteur est étonné de voir une illustration de Carl(sic) Marx, en... 1872.

1. Cet article a été réalisé dans le cadre de recherches pour une Histoire littéraire du Québec, par Laurent Mailhot, à paraître. 
Ces tiraillements sont surtout apparents pendant la première des deux grandes périodes de l'histoire du journal, période qui s'étend de 1870 à 1874 inclusivement. À cette époque, très dynamique au plan politique, l'Opinion publique intègre la production littéraire à sa vision du monde québécois. Cette réalité s'exprime clairement dans un éditorial signé par la direction.

Nous avons cru qu'un bon moyen de récompenser le public de son patronage, était d'ouvrir notre journal à tous ceux qui ont du talent et qui veulent travailler. (...) Il est trop vrai déjà, malheureusement, que le pays est ingrat pour ceux qui ont du talent, surtout pour la littérature et la poésie. (...) Pourquoi serait-il plus permis de refuser du travail à l'homme de lettres, qui vit du produit de son intelligence, qu'à l'ouvrier qui gagne son pain avec ses bras $(31 / 8 / 1871)$ ?

À partir de 1875, faisant face à de graves problèmes financiers, voulant avant tout plaire à sa clientèle, la direction évite le plus possible les prises de position politiques. Le journal devient d'abord «artistique». Mais le désengagement politique va avoir des répercussions néfastes sur l'aspect culturel. Le journal «essentiellement politique et littéraire» devient une sorte de courrier de famille... L'éditorial du nouveau rédacteur en chef est clair:

Quant au genre du journal, à la nature des articles, le changement sera radical; la politique militante, les articles de polémique seront exclus, et remplacés par un courrier exact et impartial des événements; l'Opinion publique deviendra essentiellement une publication artistique et littéraire $(4 / 1 / 1875)$.

La littérature perd de son impact en apparaissant d'abord comme un divertissement, coupé des réalités socio-politiques. Nous y reviendrons plus en détail lorsque sera abordée la partie spécifiquement littéraire du journal.

Pendant ses treize années d'existence, la première page, qui est la page éditoriale, réflète ces différentes tendances. Jusqu'en 1875, elle offre aux lecteurs un éventail de textes fidèles à sa devise: critique politique et critique littéraire, poèmes et textes sur la langue, etc. Quant aux «Portraits», ils font office d'éditoriaux. Il s'agit de biographies commentées, toujours valorisantes, au début exclusivement d'hommes politiques comme George Brown, Chauveau, Louis-Joseph Papineau, Louis-H. Lafontaine, Mgr Bourget. La longueur varie en fonction de l'importance historique. S'il y a une page pour George Brown, il y en a deux et demie pour Papineau. C'est habituellement Laurent-Olivier David qui les rédige, dans un style on ne peut plus allégorique. Pour Papin par exemple: «De ses longs bras il semblait capable d'étreindre la foule, et des éclats de sa voix, il ébranlait les maisons» $(24 / 8 / 1871)$.

Peu à peu des figures culturelles s'ajoutent à cette galerie: Philippe Aubert de Gaspé père, Madame Albani, l'abbé Casgrain, Calixa Lavallée, Crémazie, Lemay, Fréchette. 
Pendant cette période, les éditoriaux sont écrits par trois personnes: Joseph-Adolphe Mousseau, Laurent-Olivier David, Oscar Dunn. Le premier, Mousseau, co-fondateur du journal et éditorialiste de la première heure, est un homme conservateur. Il défend la Confédération et, dans l'affaire Guibord, prend fermement parti pour l'Église. Il est nationaliste avec modération.

Nous ne sommes pas de ceux qui voudraient mettre la nationalité partout, compliquer chaque question d'une autre question de race. (...) Il n'y a pas lieu dans notre pays pour une race de craindre d'être opprimée par l'autre. Nous vivons dans un pays libre où la concurrence est ouverte à tout le monde et où la palme est surtout décernée aux plus capables $(29 / 1 / 1870)$.

Ménageant la chèvre et le chou, Mousseau mêle complication et complexité. Sa naïveté fait sourire aujourd'hui.

Tous ces immensès projets, qui préoccupent l'opinion publique et font tressaillir les spéculateurs, nous inspirent de superbes considérations sur l'avenir et de beaux sentiments de patriotisme à ceux qui comptent sur l'achèvement et la permanence de l'édifice fédéral (21/7/1870).

Naïveté qui n'est égalée que par ses cris du coeur. Découvrant avec surprise que les pouvoirs du Bas-Canada risquent d'être diminués au profit du HautCanada, il s'insurge et invite toute la population à le suivre: «Notre province peut tolérer l'indépendance, souffrir l'annexion, mais supporter la domination du Haut-Canada, jamais!!! (8/9/1870). Cependant, en fédéraliste responsable et obéissant - on prend vite le pli - il se calme tout en mettant en garde le gouvernement central.

La province de Québec (...) résistera par tous les moyens en son pouvoir. C'est précisément cela qu'il fallait dire et sans ambages (...) la sentence de M. McPherson et Gray met en danger l'existence de la Confédération. Tout commentaire devient inutile (8/12/1870).

À la fin de l'année 1870 donc, un ardent défenseur de la Confédération demande un fédéralisme renouvelé... Si la position des fédéralistes est stagnante, celle des nationalistes n'a pas tellement évolué non plus. C'est ce qu'il est possible de conclure à la lecture des éditoriaux de Laurent-Olivier David et Oscar Dunn.

Rédacteur en chef de l'Opinion publique jusqu'à la fin de 1873, David marque fortement le journal dans ses premières années.

À partir de cinq variables, la psychologie des peuples, l'histoire, l'éducation, la pénurie d'entrepreneurs et la mauvaise utilisation des capitaux, David décrit et explique les maux dont souffre la société francophone. ${ }^{2}$

2. Beaulieu, André et Jean Hamelin, la Presse québécoise, II, PUL, 1975, p. 147. 
Le rêve de David est de regrouper les francophones dans un grand parti qui défendrait leurs intérêts.

Quelles que soient les divergences d'opinion des Canadiens français sur la solidité et l'efficacité de cet édifice politique, le patriotisme leur fait un devoir de tirer parti des droits qu'on nous y a laissés (19/2/1870).

Fermement opposé à la Confédération au départ, il est prêt à laisser le nouveau système faire ses preuves.

Entre les provinces anglaises qui voudraient nous écraser et les ÉtatsUnis qui nous tendront la main, la situation sera difficile pour ceux qui chercheront à conserver l'ordre actuel des choses. (...) La Confédération aura, peut-être, eu un bon effet en nous faisant comprendre la nécessité de pousser à tout prix, le Bas-Canada dans la voie du progrès $(22 / 12 / 1870)$.

Comparé à Mousseau, David est beaucoup plus efficace. Il a plus de style et ses idées sont plus claires. Si ses textes sur la France font parfois sourciller - Napoléon III n'était pas tout à fait un «symbole de démocratie» — il ne manque pas de subtilité en ce qui concerne la politique nationale. Sa position face à l'Église est étonnante pour l'époque.

Je l'ai déjà dit et je le répète (...) la religion catholique doit être douce et conciliante en ce pays; c'est la condition indispensable de sa conservation, de son influence et de sa propagation $(23 / 2 / 1871)$.

S'il fustige les journaux ultramontains, il sait aussi se montrer conciliant, car il n'est pas sans connaître le rôle de l'Église et il a besoin de son appui pour promouvoir ses idées, neuves à l'époque, que ce soit au sujet de l'éducation, où il préconise une réforme en profondeur, ou dans l'industrie.

Le Bas-Canada est fait pour être manufacturier et non pas uniquement agricole, son climat, ses pouvoirs d'eau, ses productions et ses richesses minières, tout l'indique et l'établit clairement (1/6/1871).

Ses prises de position, même face au fédéralisme, dénotent une ouverture d'esprit qui n'a rien à voir avec un nationalisme fanatique, ce qui lui vaudra d'être rejeté parfois à la fois par les conservateurs et les radicaux. Le 25 avril 1872, il s'intéresse dans son éditorial à l'Indépendance canadienne. À la lumière des démarches constitutionnelles des deux dernières années, le texte est d'actualité.

Pouvons-nous entrer dans l'indépendance, comme nous sommes, sans modifier la constitution qui nous régit depuis cinq ans? Nous croyons que non.

Il faudrait auparavant ou en même temps assurer davantage l'autonomie des provinces et l'indépendance de leurs institutions en faisant une délimitation plus parfaite du pouvoir fédéral et local. (...) 
Nous pouvons sous le régime actuel trouver dans l'Angleterre un tribunal ou un arbitre impartial dans la plupart des cas; (...). Mais où trouverons-nous ce tribunal impartial dans la Confédération devenue indépendante, entourés que nous serons de provinces et de majorités hostiles? (...) Restreindre et bien définir la juridiction et les pouvoirs du Gouvernement Fédéral et de la Cour Suprême, de manière à assurer notre indépendance absolue dans l'exercice de nos droits et la jouissance de nos institutions religieuses et nationales, est donc pour nos hommes publics un acte de sagesse et de nécessité. Ce n'est pas seulement une question nationale, c'est encore une question politique; le sentiment ici est d'accord avec la raison.

Peu à peu, ses positions se radicalisent. Des patriotes de 1837 jusqu'au cas Riel alors au coeur de l'actualité, David aborde tous les thèmes nationalistes, devenant de plus en plus cinglant.

Il est entendu que pour un bon nombre de nos capitalistes, le BasCanada est un comptoir, une espèce de table à cartes où l'on ne cherche qu'à jouer vite et hardiment pour aller jouir ailleurs (...). Ne comprendrons-nous pas enfin l'importance d'arracher le Bas-Canada au monopole qui l'écrase, de remplacer le règne de la spéculation par celui de la production, de faire en sorte que tout dans notre mouvement financier tende vers le développement des ressources de notre pays, de son industrie nationale $(11 / 6 / 1873)$ ?

Les idées exprimées dans le journal ressemblent de plus en plus à celles qui sont prônées par le nouveau Parti national fondé par de jeunes libéraux. C'est sans doute, au moins en bonne partie, pour mettre fin à cette ambiguïté que David quitte l'Opinion publique fin 1873. Il y reviendra à la fin des années soixante-dix mais pas pour longtemps. La nouvelle politique rédactionnelle ne lui permet plus de s'exprimer aussi radicalement. Après le déclenchement d'une polémique causée par un de ses articles sur l'Église, il quitte définitivement le journal. Mousseau est parti et c'est Oscar Dunn, âgé de moins de trente ans, qui le remplace comme rédacteur en chef. Il est étonnant que la direction lui ait confié ce poste. Il est le digne successeur de David qui, lui, commençait à déplaire à la direction à cause de ses positions trop tranchées.

Si Dunn a les mêmes idéaux nationalistes, sa critique de la vie politique française est beaucoup plus pénétrante. $\grave{A}$ une époque où personne au Québec n'ose contester les vingt années de pouvoir de Napoléon III, l'analyse qu'il fait de son règne est éclairante.

Napoléon III, issu de la révolution de 1848, a étouffé en 1851 le mouvement populaire qui l'a élevé. Ses panégyristes l'ont appelé l'organisateur de la démocratie: le mot est exact si la démocratie est satisfaite d'une organisation qui a pour formule le despotisme et Sedan pour terme. À vrai dire Napoléon III n'a rien organisé; il a su 
contenir durant vingt années le désordre à l'état latent, et le 4 septembre, lorsque sa déchéance a été prononcée, il est tombé victime du propre principe libéral qui avait favorisé ses débuts et son installation aux Tuileries (...) L'idée impérialiste, c'est l'idée révolutionnaire; il n'y a que l'instinct de la conservation qui fasse des Bonapartes des hommes d'ordre (1874).

Au niveau national, il prend la défense de Riel à maintes reprises, incite l'Angleterre à ne pas se mêler de la vie politique canadienne, prône une réforme dans l'éducation. La France demeure la maison-mère, alors qu'il préconise une augmentation de l'immigration française pour soutenir notre littérature...

Profession de foi, justification de son travail? Le 17 décembre 1874, son dernier éditorial s'intitule «Du style». Si le public québécois «ne regarde pas à la forme» et si «l'on peut sans logique ni grammaire, il est vrai, devenir député», il refuse de se laisser aller à la facilité et au découragement.

Aussi bien ceux qui prétendent au style doivent-ils se désintéresser du sentiment public à leur égard et, sans renoncer à se faire comprendre de la masse, ne rechercher en réalité que le suffrage d'une élite.

David et Dunn avaient du style. Efficaces, ils savaient en général aller à l'essentiel. Le principal reproche qu'il serait possible de leur faire a trait à leur notion de nationalisme. À qui s'adressent-ils? Socialement, où doit-on les situer? Sur qui, sur quoi s'appuient-ils? Si l'unité est possible dans certains cas d' 'urgence nationale», et encore, il serait illusoire de croire qu'on peut rallier une population entière au nom de la bannière nationale. La question se pose encore aujourd'hui. ${ }^{3}$ Il est à se demander si les choses ont beaucoup évolué. Les éditorialistes de l'Opinion publique avaient su, déjà, mettre à nu les principaux écueils du système dans lequel le Québec venait d'être plongé.

\section{LANGUE ET LITTÉRATURE}

Si 1875 marque la fin d'une époque glorieuse au plan politique, c'est, semble-t-il, au profit d'une orientation plus culturelle. Malheureusement ce renouveau ne durera qu'un temps, et le journal va plutôt s'affadir. L'augmentation quantitative des textes de fiction ne signifie pas grandchose. La place de plus en plus grande prise par les textes français, par

3. Le congrès péquiste de décembre 1981 en est un exemple typique. Au lieu de créer une discussion, il a provoqué la panique de ses dirigeants. Cette attitude perpétuait le cafouillage référendaire, attitude qui laissait croire que toute discussion en profondeur était «néfaste à la cause». Il est aberrant de constater que les tenants du «oui» affirmaient que rien n'allait changer advenant la souveraineté, alors que les tenants du «non» affirmaient que tout allait changer s'il ne se passait rien... À défaut de cadre théorique solide pour se situer, il est illusoire de vouloir convaincre les gens. Si on affirme que rien ne va changer, pourquoi les gens risqueraient le changement? Cent ans après l'Opinion publique, le fond du problème reste le même. 
exemple, ne doit pas cacher le fait que, en revanche, la proportion des textes québécois diminue considérablement. Ce qui semble être un désintérêt pour la littérature québécoise est confirmé par le fait que le nombre de textes critiques diminue de près de $40 \%$ entre 1875 et 1882 . D'autant plus qu'on compte beaucoup de reproductions dans ce qui reste: discours, témoignages. L'Opinion publique n'est plus un chantier mais une simple vitrine. Le changement ne sera pas brutal cependant, et même dans les pires années (1877-78), il reste encore de nombreux textes intéressants. C'est surtout l'unité du journal qui y perd.

C'est l'écriture même qui fait d'abord l'intérêt du journal. La fiction - poème, feuilleton, nouvelle - n'en est qu'un aspect. Il faut voir l'Opinion publique comme un journal d'écrivain.

Entre avril et juillet 1875, Benjamin Sulte, collaborateur régulier, publie une série d'articles intitulée «Vieilles gazettes», sur l'histoire des journaux au Bas-Canada. Plus qu'une étude historique, c'est celle de l'écriture au Québec. Quand, au début des années 1870, un incendie dévasta Chicago, le reportage - en six parties - est confié à Fréchette. Inutile de dire que ça n'avait rien de factuel...

L'intérêt marqué pour ce drame montre bien que le journal, malgré ses tendances nationalistes, n'est pas limité au monde québécois. Les «correspondances de voyage» sont particulièrement intéressantes et ne ressemblent en rien à celles que nous connaissons aujourd'hui, que ce soit les notes de Buies sur son voyage à San Francisco - où on apprend que «le saucisson est antipathique aux relations sociales» - ou les «Lettres américaines» de Casgrain et Marmette, notes très personnelles où on affirme qu'Edgar Allan Poë serait mort assassiné et non alcoolique.

Cette version (l'alcoolisme), trop accréditée, n'est qu'une calomnie propagée par les ennemis de Poë ou par des esprits enclins à adopter les assertions du vulgaire, s'ingérant de condamner sans rémission, sur les apparences, des esprits supérieurs comme l'auteur du Corbeau (9/3/1882).

La direction n'a jamais caché sa volonté de publier les meilleurs écrivains québécois et encourageait ceux qui voulaient écrire.

Nous comptons sur plusieurs (...) excellents écrivains, dont les uns ne nous ont pas encore donné de réponse formelle et les autres préfèrent conserver l'incognito pour le présent. Nous invitons cordialement la jeunesse instruite et studieuse à nous envoyer des essais littéraires de tout genre. Nous leur ferons bon accueil et publierons les pièces les plus méritoires $(3 / 2 / 1876)$.

Un mois plus tôt, la direction mettait en première page la liste de ses collaborateurs: Casgrain, Evanturel, Fabre, Fréchette, Lemay, Marmette, Sulte... 
Dans les premiers temps, la littérature envahit tout. Ainsi Ferdinand Gagnon, qui rédige une chronique sur les États-Unis, a trouvé la solution miracle pour rapatrier les colons: leur faire lire Jean Rivard.

Voilà un manuel de défrichement(sic), et un joli traité de patriotisme en action. Chaque phrase est un encouragement pour le colon, et chaque ligne est un petit poème qui chante les beautés de la vie champêtre (...). Je crois fermement que Jean Rivard devrait être le premier et le plus effectif des moyens à prendre pour opérer un rapatriement des colons. Ce livre vaut mieux que toutes les brochures possibles (22/10/1874).

L'importance de la lecture explique celle donnée aux nombreux articles sur la langue et l'éducation. Ils sont, au cours des premières années surtout, éminemment politiques.

Donc, suivant nous, la cause de notre abrutissement intellectuel est inhérente à notre situation politique; l'effet qu'elle produit se nomme découragement et non ineptie, suffisance, paresse (Edmond Lareau; $16 / 2 / 1871)$.

Le sujet préoccupe. Un éditorial intitulé «Abrutis par les livres», en 1870, cause une polémique sur l'éducation, la lecture, le choix des livres. C'est dans ce contexte que David ébauchera son programme:

Pour que l'éducation fasse le bien qu'elle poursuit dans le monde, il faut qu'elle soit en rapport avec les destinées, les aptitudes, les besoins et les exigences des peuples, qu'elle donne à toutes les classes de la société les connaissances nécessaires à leur avancement, à leur bonheur et à leur progrès $(2 / 2 / 1871)$.

Au milieu de débats politico-linguistiques - en 1880 un éditorialiste exhorte ses concitoyens à écrire en français à Ottawa et enjoint les Anglais à apprendre le français - on donne la «Liste alphabétique des barbarismes les plus répandus parmi nous», liste qui aurait fait les délices de Léandre Bergeron. On y apprend qu'un «snack» est un «régal», qu'un «bed» est un «lit de camp» et que pour «garde-z-yeux» il faut aller voir à «oeillère». Les études sur la langue sont nombreuses et variées, au point de présenter un lexique de la langue iroquoise.

L'évolution de l'éducation en France est suivie de près et pas toujours de façon progressiste, surtout en ce qui concerne l'éducation des femmes.

La femme, c'est ce qui, en ce moment, se prête le moins à la réforme. (...) Les deux systèmes d'éducation de la femme sont bien connus par leur résultat. Le nôtre a produit la religieuse, l'humble soeur et le vôtre ne peut produire que des monstres comme les sanglantes tricoteuses de 93, admiratrices de la guillotine et les pétroleuses de la Commune $(2 / 2 / 1882)$. 
De la France, encore plus que sa politique ou son système d'éducation, c'est sa littérature qu'on scrute. "Nous sommes heureux d'apprendre que notre nouveau feuilleton illustré est très aimé de nos lecteurs. Ce n'est pas étonnant, car ce roman a fait fureur en France» (6/1/1882). Signe indiscutable de qualité? En tout cas, signe qui montre l'importance que l'on accordait à ce qui venait de France. L'opinion de Victor Hugo est toujours prise en considération, qu'on soit pour ou contre.

Victor Hugo a toujours eu des prétentions à jouer un grand rôle politique et il n'est pas douteux que l'idée politique ait exercé sur son immense talent littéraire et poétique une influence funeste, dont les effets se sont surtout fait sentir dans les Travailleurs de la mer et dans l'Homme qui rit (30/5/1872).

Trois quarts de siècle plus tard, Roger Duhamel inversera le jugement dans le Devoir, en écrivant que c'est sa grande imagination qui a joué un rôle funeste sur ses idées politiques.

En général, la tendance est conservatrice. Dans un article, on met dans le même sac Zola, Hugo, Eugène Sue, Garibaldi, Gambetta, les communards, tous condamnés en tant qu'«impies». Il n'y a pas que les Français, d'ailleurs, qui subissent les critiques: «Il paraît que le monde littéraire va être affligé des Mémoires d'Oscar.Wilde et d'un récit de son voyage en Amérique. Nous ne lui avons pourtant fait aucun mal» (19/10/1882). Par contre, paradoxalement, Voltaire n'est pas trop mal vu. On parle même de Chateaubriand comme du "Voltaire catholique».

Les grands écrivains sont Déroulède, Sully-Prudhomme, Alphonse Daudet. Aucun qualificatif ne semble assez fort pour décrire l'oeuvre du poète Autran qui vient de publier le 8ème et dernier volume de ses oeuvres complètes. «Ses Poèmes de la mer sont une oeuvre absolument personnelle, absolument originale, qui mérite de vivre et qui vivra» $(18 / 8 / 1881)$. Si le journal se trompe en ce qui a trait à Autran, il a vu juste pour Edgar Allan Poë, dont on publie Double assassinat des plus singuliers en 1870.

Évidemment, on préfère que les Français parlent des Québécois. C'est avec joie qu'on reproduit le texte de Raoul Fray intitulé «Le Canada français et sa littérature», ou celui de la conférence du consul Lefaivre qui traite de nombreux écrivains: Crémazie - le meilleur selon lui -, Lemay, Fréchette, Chauveau, Marmette, Faucher de Saint-Maurice, Étienne Parent.

Les intellectuels français ne sont pas tous aussi curieux. En réponse à une lettre, écrite à propos d'un article où il était question des races et d'où les Québécois étaient absents, Littré écrit: «Dans mon article, en parlant de la France, j'avais bien dit qu'elle avait une annexe au Canada, mais j'ignorais que cette annexe fût si importante» $(17 / 7 / 1879)$. 
"L'annexe» se manifeste en se faisant publier en France. C'est le cas de Gérin-Lajoie qui publie Jean Rivard en feuilleton dans le journal le Monde. C'est surtout le cas de Fréchette qui remporte un prix de l'Académie française. Le succès est tellement grand que toutes les semaines, de novembre 1878 à février 1879, l'Opinion publique publie les lettres de félicitations que l'écrivain reçoit, dont celles de Dumas fils, Hugo, Leconte de Lisle, Sully-Prudhomme... Pendant les treize années d'existence du journal, cet événement est sans conteste le plus marquant en ce qui concerne l'impact de la littérature québécoise en France.

Il est étonnant, dans un pays où la littérature est aussi jeune, de voir l'intérêt avec lequel on vise à se doter de structures. D'une part l'importance accordée aux structures académiques est claire. Le respect prodigué à l'égard de l'Académie française ne fait pas de doute et les articles faisant la promotion d'une académie locale sont éloquents. Les écrivains se plaignent en général du manque d'institutions littéraires.

On avait autrefois deux ou trois institutions littéraires où des lectures et des discussions des plus intéressantes formaient la jeunesse et répandaient parmi le peuple des connaissances et des sentiments utiles. Maintenant on n'a plus que des réunions politiques et des clubs de cartes. Peut-on dire que nous avons progressé? (1/5/1879)

Beaucoup plus intéressante est la primauté que l'on accorde à la critique et à l'histoire littéraire. Ces deux sujets constituent la base d'articles de fond comme «Notre passé littéraire et nos deux historiens» de Casgrain (8/6/1882), "Le Canada français et sa littérature» de Sulte (série d'articles qui vont de la fin de 1878 au début de 1879), "Quelques considérations sur la littérature et les beaux-arts dans la province de Québec» de Napoléon Legendre (5/10/1876 et 12/10/1876). Quant aux deux articles de Casgrain publiés en août 1872 et intitulés "Critique littéraire», ils développent un sujet qui ne semble malheureusement pas avoir été bien compris, celui de la nécessité d'avoir des critiques solides et suffisamment objectives en regard de la production locale.

La littérature américaine, qui date d'hier comme la nôtre, a acquis cette virilité qu'on lui connaît, précisément en donnant à la critique ses coudées franches, en laissant aux juges littéraires le même franc parler qu'ils ont en Europe. Le temps est venu, croyons-nous, d'agir avec la même liberté, d'apprécier nos écrivains non pas à la valeur relative mais à la valeur absolue (15/8/1872).

Ces écrivains dont parle la critique sont publiés dans l'Opinion publique. Ce travail de création québécoise, tant au niveau du texte que du métatexte, s'amenuisera peu à peu après 1875 , touchant le creux de la vague en 1877-78. Les textes français prennent de plus en plus de place, les textes critiques diminuent (pour des statistiques plus précises, voir le tableau en annexe). 
Le journal a ses «poètes-maison». De loin le plus populaire, William Chapman, avec plus de soixante poèmes, devance Louis-Honoré Fréchette (trente textes). Parmi les autres poètes québécois fréquemment publiés, il faut surtout mentionner les noms de J.-Adolphe Poisson, Emmanuel Blain de Saint-Aubin, Nérée Beauchemin. Les poètes français qu'on y lit ne sont pas nécessairement les plus intéressants: Charles-Perotte Deslandes, Eugène Manuel. Aucun n'y publie plus d'une dizaine de poèmes. Pour ce qui est de la prose, aucun nom ne se détache nettement, tant chez les Québécois que chez les Français.

Avec environ 25 textes touchant à sa vie ou à son oeuvre, LouisHonoré Fréchette se place loin devant Octave Crémazie et Victor Hugo. Le nom d'Arthur Buies est mentionné souvent, même s'il n'a pas très bonne presse à l'époque.

$\mathrm{Si}$ les feuilletons sont toujours français, le lecteur trouve par contre dans le journal des poèmes anglais en traduction locale. C'est le cas du Prisonnier de Chillon de Byron (28/12/1871) ou d'un texte de Longfellow en 1882, accompagné d'un article sur ses traductions au Québec.

On peut faire la moue devant les chroniques de livres - «Bibliographie» ou «Petite Revue artistique et littéraire» - qui servent surtout à présenter des livres comme "Une mine de pensées détachées à l'usage des cultivateurs par le révérend $\mathrm{P}$. Lacasse», il reste que l'Opinion publique a su faire une place de choix à la littérature québécoise.

Encore aujourd'hui, c'est un journal qu'on lit et qu'on regarde avec plaisir. Les gravures sont toujours très belles, illustrant aussi bien des scènes de la vie québécoise que des événements ou des personnages historiques, ou des animaux dans leur habitat naturel.

L'Opinion publique a dû disparaître parce qu'elle n'a pas su s'adapter à ses propres transformations. Voulant «exclure entièrement (...) les discussions politiques et les attaques personnelles", la revue aurait peut-être pu devenir un bon organe culturel. Dynamique en 1875, intégrant de nombreuses nouvelles chroniques, il était possible de le croire. Mais elle ne pourra, en définitive, choisir une voie précise. Culturelle mais négligeant de plus en plus le cadre théorique, continuant malgré tout à parler de politique mais de façon ennuyeuse, se rétractant lorsque les positions prises par un journaliste ne sont pas assez neutres, incapable d'imposer une certaine continuité aux chroniques, la revue deviendra plutôt un espèce de fourre-tout, un salmigondis inconsistant. À force de vouloir plaire à tout le monde, l'Opinion publique finira par ne plus rejoindre personne.

La direction le sentait déjà en 1879 , elle qui souhaitait à ses abonné(e)s, pour la nouvelle année: "Puissent-ils aussi, dans leur intérêt comme dans le nôtre, continuer à lire l'Opinion publique et à le payer, le payer surtout, s'ils veulent être heureux en ce monde et dans l'autre». 


\section{ANNEXE: PLACE DE LA LITTÉRATURE}

1870 (le journal a seulement 8 pages)

poésie: 14 textes au total

textes québécois: 10 ( $71 \%$ app.) textes français: 4 (29\% app.) autre: 0

prose (nouvelle, conte, feuilleton): 10 textes

textes québécois: $6(60 \%)$ textes français: $3(30 \%)$ autre: $1(10 \%)$

critique/autre (biographie, essai, témoignage): 4

1875 (le journal a 12 pages)

poésie: 19 textes

textes québécois: $8(42 \%)$ textes français: $9(47 \%)$ autre: $2(11 \%)$

prose: 25 textes

textes québécois: $12(48 \%)$ textes français: $10(40 \%)$ autre: $3(12 \%)$

autre/critique: 40 textes

1882 (12 pages)

poésie: 49 textes

textes québécois: $17(34 \%)$ textes français: $27(53 \%)$ autre: $5(13 \%)$

prose: 26 textes

textes québécois: $3(11.5 \%)$ textes français: $20(77 \%)$ autre: $3(11.5 \%)$

critiquelautre: 25

poésie québécoise de 1870 à 1882 : baisse de $36.3 \%$

poésie française pendant cette période: hausse de $24 \%$

prose québécoise de 1870 à 1882: baisse de $48.5 \%$

prose française pendant cette période: augmentation de $47 \%$

textes critiques de 1870 à 1882: baisse de $37.5 \%$ 